\title{
Cibi di pace, cibi di guerra: la storia dell'alimentazione al liceo
}

\section{La preparazione preliminare, le finalità, gli strumenti}

Lo spunto del progetto didattico Cibi di pace, cibi di guerra, rivolto nell'anno scolastico 2015-2016 alla quinta ESABAC del Liceo Linguistico "Laura Bassi" di Bologna, è nato dalla partecipazione della classe, assieme alla docente di lettere e storia, alla presentazione, il 4 novembre 2015, del portale Guerrainfame. La guerra non è mai servita. Cibi di guerra, strategie e politiche alimentari nella prima metà del Novecento in Italia, a cura degli Istituti Storici dell'Emilia Romagna in Rete, coordinati dall'Istituto per la Storia e le Memorie del '900 Parri E-R. Considerata la predisposizione nel sito di una sezione destinata ai Progetti didattici, si è pensato di realizzare attività ispirate alle suggestioni e agli stimoli culturali nati dall'indispensabile preparazione preliminare delle conferenze al Parri. L'idea era di costruire un percorso interdisciplinare volto a presentare una storia del costume italiano - e fin dove possibile europeo - attraverso abitudini e necessità alimentari nel periodo 1880-1950.

Tenendo conto sia delle peculiarità del percorso di studi specifico degli studenti', sia della metodologia suggerita dal progetto degli istituti storici emiliano romagnoli, si è mirato a una verifica diretta delle informazioni ricevute dagli esperti, attraverso la disamina di testimonianze raccolte personalmente, dirette (orali) e indirette (letterarie), puntando ad un'interazione tra didattica delle conoscenze e didattica delle competenze. Come seconda finalità il progetto doveva promuovere le capacità di team working, oltre che di rielaborazione personale, in una 
condivisione di studi ed esperienze professionalizzante, ma anche propedeutica a percorsi di ricerca post-liceali. Fasi di lavoro individuale sono state alternate ad altre di gruppo, avvalendosi per la condivisione della documentazione, per la sua correzione e per la compartecipazione dei risultati di una piattaforma web in comune tra alunni e docente.

\section{Le fasi di realizzazione del progetto}

Il progetto è stato concepito sin dall'inizio come work in progress, ovvero "ricerca aperta" agli stimoli provenienti dalle "scoperte" compiute, ma anche alle curiosità e alle proposte degli studenti. Facendone oggi un consuntivo, se ne possono identificare sette momenti/aspetti distinti.

- Il presupposto è stato la conoscenza di alcuni aspetti fondamentali del tema del cibo e dell'alimentazione dal periodo della «guerra dei trent'anni 19151945» agli anni Cinquanta. Pertanto gli studenti hanno seguito, oltre alle conferenze del 4 novembre, anche quelle della giornata di studio del 26 febbraio 2016 su Cibi di guerra: vita quotidiana, produzione e consumi in Italia (1915-1945), e ne hanno trascritto gli appunti sulla piattaforma web della classe, in modo che tali documenti potessero essere integrati e corretti da tutti e costituire il punto di riferimento per ogni gruppo di lavoro. Alcuni studenti si sono documentati sullo specifico bolognese con la lettura dell'opera miscellanea di Goldoni, Ferrari e Leoni (eds.) 1980, e in particolare dei capitoli Quando la dieta era più ferrea della volontà e E fu subito pane nero (Ferrari 1980 e Soglia 1980).

- Dopo la prima giornata di studi, si è tentato di verificare - sia pure in scala ridotta e di necessità memorialistica - alcuni dei dati presentati dai relatori. Gli studenti hanno intervistato gli anziani di casa - i nonni, principalmente con un questionario da loro elaborato sulla base delle curiosità indotte dalle conferenze e mirato a conoscere le abitudini alimentari nel periodo intorno alla Seconda guerra mondiale.

- In alcuni casi, oltre a trascrivere le risposte, gli studenti hanno anche filmato o registrato l'intero colloquio, da cui si potevano evincere altre informazioni sul modo di vivere nel periodo in oggetto. Le interviste sono state analizzate con l'obiettivo di ricavarne delle statistiche sulla traccia di sei temi-guida suggeriti dall'insegnante (individuati incrociando le spiegazioni degli specialisti con le risposte degli intervistati): gli studenti, divisi in gruppi, hanno riesaminato la documentazione per verificare se si potessero individuare analogie o differenze significative nelle risposte, a seconda dell'età, del sesso, 
della zona di provenienza, del tenore di vita, e per compilare infine un quadro riassuntivo (anche in forma di grafici e tabelle) delle informazioni ricevute sull'alimentazione e sulle esperienze più marcanti dei loro familiari nel periodo tra la Seconda guerra mondiale e l'immediato dopoguerra.

- Alcune studentesse, ispirate dai documenti pubblicati sul portale Guerrainfame, hanno pensato di averne un riscontro testimoniale intervistando le loro nonne: ne sono nati due «ricettari di memoria», che hanno la peculiarità di corrispondere l'uno all'esperienza dell'Italia settentrionale e l'altro a quella dell'Italia meridionale e di segnalare la disponibilità dei diversi alimenti su base geografica.

- Nel frattempo, si è cercato di orientare al tema approfondito anche le letture assegnate ogni mese alla classe nello studio della letteratura italiana: in particolare, dai libri Sull'Oceano di Edmondo de Amicis e Niente di nuovo sul fronte occidentale di Erich Remarque sono state tratte per iscritto delle "antologie" tematiche commentate, presentate da un'introduzione generale e condivise sulla piattaforma web di classe, mentre La pelle di Curzio Malaparte e Ragazzi di vita e Una vita violenta di Pier Paolo Pasolini, nonché altri documenti storico-letterari, sono stati analizzati oralmente in classe, con l'obiettivo di mettere in luce le trasformazioni dalla fine dell'Ottocento in Italia (De Amicis), alla Prima guerra mondiale in Germania (Remarque), alla fine della Seconda guerra mondiale attraverso la Penisola (Malaparte), al secondo dopoguerra a Roma (Pasolini).

- Una delle studentesse ha scelto di completare il percorso in chiave interdisciplinare presentandolo come "tesina" all'esame di maturità (G. Bernardi, Il problema dell'alimentazione dalle due guerre mondiali ai giorni nostri), in cui ha confrontato la penuria alimentare delle epoche passate con la sovrabbondanza e gli abusi dell'alimentazione contemporanea.

\section{I lavori di gruppo sulle interviste}

L'originalità e l'interesse del percorso sono consistiti in primo luogo nella possibilità di reperire personalmente fonti memoriali, diverse dalla documentazione pre-selezionata da specialisti. L'impatto emotivo dell'operazione ha, tra l'altro, consolidato la sensibilità e la consapevolezza dei ragazzi nei confronti del valore testimoniale di esperienze individuali e collettive, e perciò della necessità di conservarle e trasmetterle, specie avvalendosi dei moderni strumenti informatici e mediatici, che offrono accessibilità e immediatezza nella condivisione e diffusione dei materiali. Gli studenti - in età tra i 18 e i 20 anni - si sono confrontati con testimo- 
nianze dirette, ciò che si è tradotto nella raccolta di una piccola e partecipata "banca della memoria" che ha permesso un dialogo intergenerazionale, esperienziale ed affettivo, con gli anziani di casa, sorpresi dall'interesse dei nipoti che rivalutava i loro ricordi e la loro esperienza, caricandoli di nuovo significato e valore. Tali testimonianze, che ci si sarebbe potuti aspettare in parte deformate o distorte nella memoria e nel racconto degli intervistati, dato l'ampio lasso di tempo trascorso dai fatti riferiti, hanno invece trovato coincidenze con quanto gli alunni avevano appreso dagli "specialisti", contribuendo non solo ad approfondire il quadro offerto dalla storiografia, ma soprattutto a convalidarlo con il marchio dell'esperienza viva (degli anziani) e dell'appartenenza e della condivisione (per i giovani).

Questo il senso dell'analisi statistica dei dati: seppur su un "micro-campione" di una ventina di interviste, verificare se e in quale misura tali racconti corrispondevano coi "dati dei libri". Al fine di meglio interpretare la natura e l'origine di tali testimonianze, pertanto, gli alunni - divisi in gruppi - hanno catalogato statisticamente il "profilo anagrafico" dei loro intervistati (età, sesso, luogo d'origine), cercando di metterne in rapporto l'alimentazione col luogo di provenienza (città/cam-

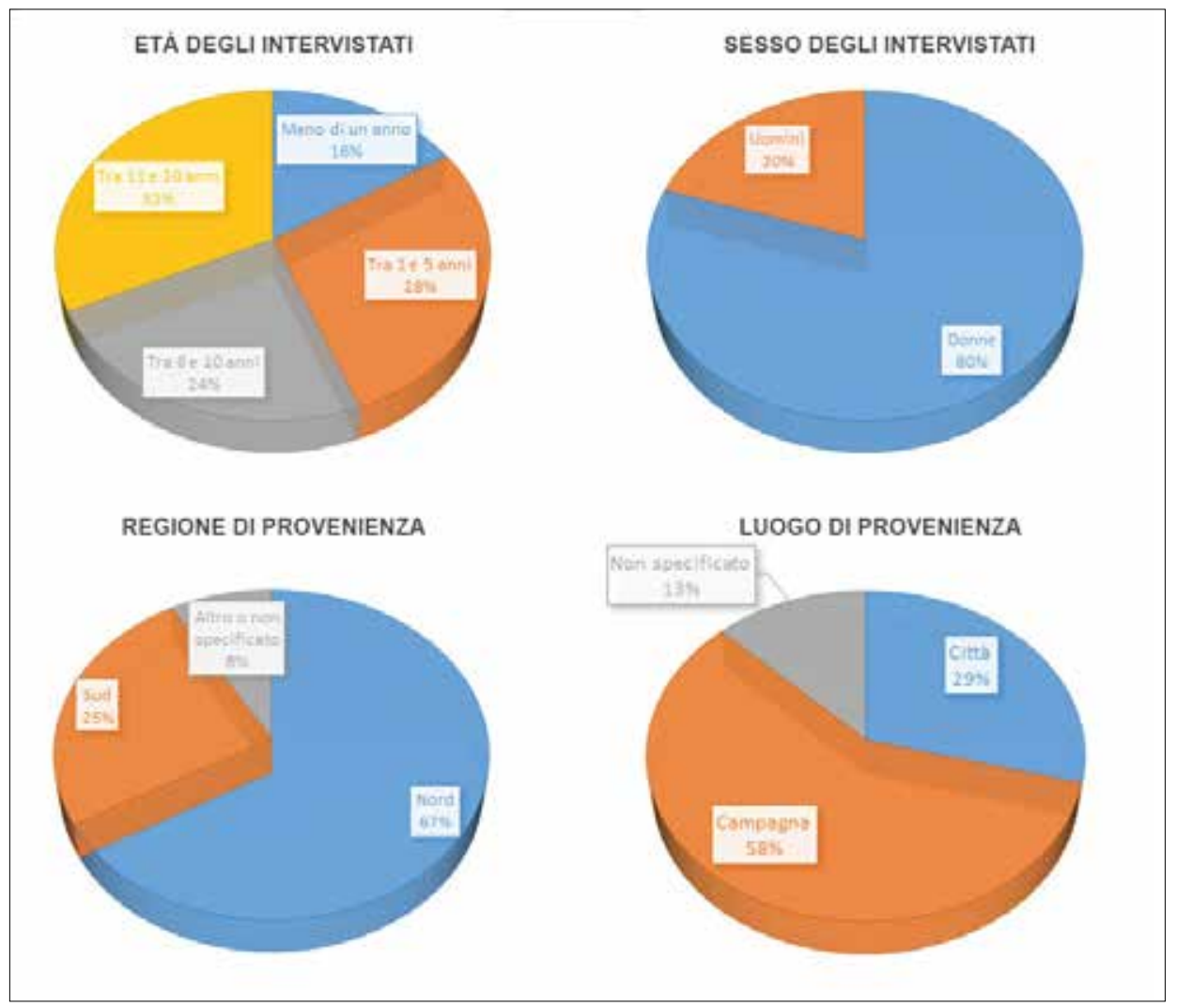

G. Magli, M. Mennillo, M. Soligo, Metodologia e dati raccolti. 
pagna; Italia settentrionale/meridionale) e con le condizioni socio-economiche. Hanno inoltre distinto i cibi di consumo quotidiano da quelli delle feste, giungendo a conclusioni piuttosto omogenee nel confronto tra città e campagna, Nord $\mathrm{e}$ Sud durante la Seconda guerra mondiale:

Anche se, in tempo di guerra, c'erano diverse discrepanze tra l'alimentazione di campagna e quella di città, la dieta era più o meno omogenea per contadini e cittadini. Era comune il consumo di pane (nero), pasta e polenta, fatti esclusivamente in casa, e quello di verdure, legumi, tuberi e frutta, soprattutto pomodori, fagioli, patate e mele (G. Boschi, A. Ciminnisi, G. Lazzari, C. Paolucci, L. Ricci Maccari$\mathrm{ni}$, Le differenze nell'alimentazione italiana tra città e campagna)?

Al nord si è registrata una presenza maggiore di padri di famiglia impegnati in attività lavorative $o$ in fabbriche, o nelle ferrovie, che fruttavano comunque un buono stipendio. Invece, al sud i contadini erano ancora molto attivi, scambiavano prodotti con altri beni, e ovviamente la loro paga dipendeva sostanzialmente da quanto il padrone concedeva. Stando ai dati raccolti, si può anche notare che gli alimenti presenti tutti i giorni nelle tavole delle famiglie italiane durante la guerra sono più o meno gli stessi, anche se alcuni di essi sono più frequenti al nord e altri al sud (C. Abbate, M. Marisaldi, M. Romeo, Le differenze nell'alimentazione italiana tra nord e sud della Penisola).

Chi abitava in campagna, pur avendo un numero di figli mediamente superiore rispetto a quelli che vivevano in città, godeva di una condizione di vita più agiata a confronto dei cittadini [...], perché chi apparteneva ad una famiglia di contadini o di allevatori poteva disporre di verdure, legumi e ortaggi freschi, per non parlare dei cereali come frumento, orzo e riso, le cui eccedenze erano di solito vendute o barattate con alimenti più difficilmente reperibili o articoli di abbigliamento (Boschi, Ciminnisi, Lazzari, Paolucci, Ricci Maccarini, cit.).

Molto dipendeva dalle condizioni economiche: «se non si aveva un reddito costante o un patrimonio familiare [...] era molto difficile mantenersi»; da queste, e dal grado di ricchezza, derivava la discrepanza più evidente nell'alimentazione degli italiani:

Nella nostra indagine le più consistenti differenze si sono riscontrate proprio nei dolci: si potevano consumare dai dolci più raffinati, come la zuppa inglese o i bomboloni ripieni alla crema, a quelli "caserecci", come la ciambella dura o la polenta dolce con ricotta, fino a quelli legati alle tradizioni locali, ad esempio i "mustazzoli" o i "purcidduzzi". Comunque tutti questi dessert erano a base di latte 
e uova, due ingredienti quasi introvabili (ibidem).

Anche il clima e la natura del territorio influivano sulle discrepanze alimentari:

Le famiglie del sud facevano meno fatica a procurarsi verdure e legumi rispetto a quelle che abitavano al nord, dove invece i derivati della farina come pane e pasta non mancavano mai nella dieta quotidiana, così come le patate e la polenta. Inoltre, al nord un alimento ricorrente è la castagna, che spesso veniva utilizzata dalle donne per fare una polenta dolce (Abbate, Marisaldi, Romeo, cit.).

Altri studenti hanno indagato sull'incidenza della pubblicità nei consumi alimentari tra fascismo e guerra, rimarcando che non era come quella odierna e si poneva sostanzialmente altri obiettivi: «allo scoppio del conflitto la televisione non esisteva ancora, mentre la radio, pur possesso solo di pochi privilegiati, fu uno dei mezzi più potenti per giustificare e promuovere, specie tra i ceti borghesi, gli ideali e la retorica fascista» (J. Awuku, M. Caminetti, L. Saiola, C. Venturi, L'importanza della pubblicità rispetto ad oggi), ideologia che era oggetto principale della propaganda. Invece, per quanto riguarda i prodotti di consumo,

I manifesti pubblicitari - che raffiguravano spesso uomini e donne che sventolavano bandiere italiane - promuovevano assiduamente i prodotti nazionali, in una sorta di "guerra nella guerra" nei confronti dei prodotti stranieri, che venivano al contrario screditati. Erano quindi molto diffuse pubblicità di marche e di prodotti tipici italiani, come FIAT, Alfa Romeo, Campari ecc., come hanno ricordato alcuni nostri testimoni che risiedevano in città (ibidem).

Peraltro, sono appunto solo i residenti nelle città a ricordare le réclames, mentre gli altri «difficilmente hanno testimonianze di pubblicità del tempo, poiché nelle campagne e nelle zone rurali qualsiasi tipo di tecnologia era praticamente inaccessibile», anche se qualche intervistato ha fatto riferimento alla "radio rurale" voluta da Mussolini ${ }^{3}$.

Un altro gruppo ha raccolto, paragonato e commentato i "ricordi più intensi" rievocati dagli anziani: si tratta in realtà prevalentemente di ricordi "ricorrenti", come l'alimentazione a base di polenta e feculacei, verso cui alcuni «mostrano ancora la loro intolleranza attuale» (G. Bernardi, C. Di Giulio, G. Lelli, A. Tabellini, Il ricordo più intenso), o come la scarsità del pane, di cui era reperibile praticamente solo quello nero, la fame per i razionamenti o la paura dei bombar- 
damenti aerei, ma anche la solidarietà e la condivisione del poco disponibile. Il giudizio su tedeschi e alleati, inoltre, è risultato fortemente condizionato dalla loro generosità: in particolare, coincidono le memorie sull'arrivo degli americani, presentati come coloro che «distribuivano provviste alle famiglie», offrendo ai bambini cioccolata, tè e biscotti e riportando sul mercato alimenti prima introvabili, come lo zucchero.

Infine, sono stati analizzati i dati relativi alle trasformazioni del dopoguerra, quando ricomparvero più alimenti di base della dieta italiana, di cui peraltro già disponevano anche in periodo bellico diversi degli intervistati residenti in campagna.

Circa il $60 \%$ degli intervistati ha vissuto nei primi anni successivi alla guerra un miglioramento quantitativo e qualitativo delle proprie condizioni alimentari grazie alla maggiore reperibilità di cibo, che ha permesso loro

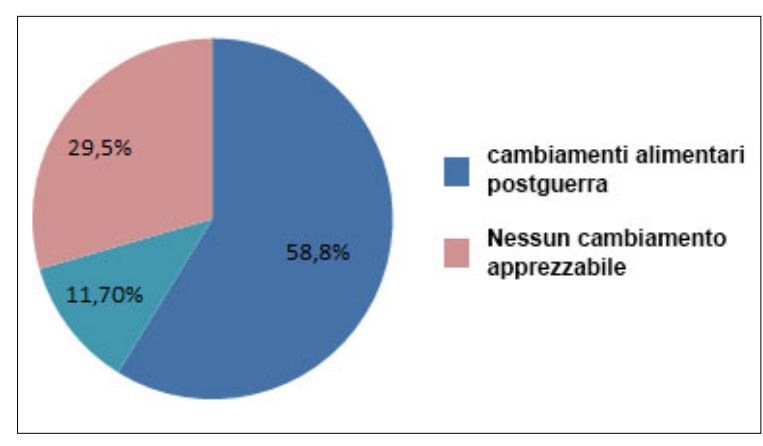

G. Magli, M. Mennillo, M. Soligo, Metodologia e dati raccolti. un consumo più consistente di cibi sostanziosi quali zucchero, carne e pane, razionati o comunque molto costosi durante la guerra (N.-T. Licciardi, A. Montebarocci, C. Tiron, R. Turco, L'immediato dopoguerra).

Esito globale della ricerca è stata la constatazione dell'influenza delle possibilità di alimentarsi sulla mentalità collettiva: ad esempio, a fondamento di una cultura del risparmio e del riciclo, ma anche di un'etica della generosità e della condivisione «che colpisce, in tempi di tante ristrettezze» (Abbate, Marisaldi, Romeo, cit.).

\section{Le antologie tematiche}

Anche in alcuni dei classici letti in versione integrale sono state ricercate ed individuate informazioni che integravano il quadro dell'alimentazione dall'età postunitaria a quella post-bellica (1880-1950 circa). In particolare, per Sull'Oceano di Edmondo De Amicis (1889) e Niente di nuovo sul fronte occidentale di Erich Remarque (1929), gli studenti hanno curato delle antologie ragionate, corredandole della sintesi dei dati raccolti e del loro commento e presentandole con una sintetica ricerca sulle condizioni di vita e di alimentazione dell'epoca descritta. 
Così, il libro Sull'Oceano - sorta di giornale di bordo in cui De Amicis, con finalità di analisi sociale, descrive un suo viaggio di ventidue giorni da Genova a Buenos Aires sul piroscafo "Galileo" - ha offerto uno spaccato delle abitudini alimentari dei viaggiatori di prima e di terza classe, riflesso del tenore di vita (e dei relativi pregiudizi), ma anche delle origini geografiche e dei rapporti interpersonali, visto che tali descrizioni si svolgono soprattutto nei momenti conviviali, quali pranzo e cena:

In tutto il testo il cibo fa comunque da cornice degli eventi, tanto più che i riferimenti ad esso indicano lo status sociale dei personaggi della storia.

Ad esempio, non tutti i passeggeri del piroscafo hanno il permesso del capitano di bere il vino, che è concesso solamente alle classi più abbienti, visto che il capo della nave teme che le persone più umili, inebriate dal liquore, possano compiere atti osceni o criminali. Ciò sottolinea il disprezzo che i borghesi dell'Ottocento provavano nei confronti del proletariato (G. Lazzari, C. Paolucci, L. Ricci Maccarini, Cibo e condizioni sociali nel libro Sull'Oceano di Edmondo De Amicis).

De Amicis offre uno spaccato della società del tempo («una varietà abbastanza soddisfacente per un osservatore»), contrapponendo efficacemente i lussi della prima classe («seduti a una tavola lunghissima, in mezzo a un vasto salone, tutto messo a oro e specchi», «[...] era tutta occupata a mangiare con coscienza, facendosi tirar giù da una di quelle credenze aeree che ci ciondolavano sul capo come lampadari, ora la senapa, ora il pepe, ora la mostarda, come se volesse raccomodarsi lo stomaco guasto e schiarirsi la voce rauca, che andava provando tratto tratto con un colpetto di tosse») alla miseria della terza classe («spettacolo [...] dove la maggior parte degli emigranti, presi dal mal di mare, giacevano alla rinfusa, buttati a traverso alle panche, in atteggiamenti di malati o di morti, coi visi sudici e i capelli rabbufati, in mezzo a un grande arruffio di coperte e di stracci [...]»; «La sola nota amena di quello spettacolo erano i pochi intrepidi che, sopra coperta, uscivan dalle cucine con le gamelle colme di minestra tra le mani, per andarsela a mangiare in pace ai loro posti: alcuni, facendo prodigi d'equilibrio, ci riuscivano; altri, messo un piede in fallo, cadevano col muso nella gamella, spandendo brodo e paste da tutte le parti, in mezzo a uno scatenamento di maledizioni»). Dal romanzo, portavoce delle speranze e delle paure dei migranti, emerge che il motivo per il quale gli italiani emigravano verso le Americhe era sfuggire alla povertà ([...] «non emigrano per arricchire, ma per trovar da mangiare ai loro figliuoli» [...]). Il divario tra la ricca alimentazione dei benestanti e la frugalità dei pasti dei migranti della terza classe si può pertanto riscontrare in molti passi del romanzo (C. Abbate, N.-T. Licciardi, M. Marisaldi, A. Montebarocci, M. Romeo, C. Tiron, R. Turco).

Ancor più fertile la riflessione sul tema dell'alimentazione a margine della lettura di Niente di nuovo sul fronte occidentale, nel quale la denuncia della crudeltà della guerra s'interseca con la rappresentazione del tragico rapporto dei soldati col cibo: 
Il romanzo si apre immediatamente con una scena drammatica: di centocinquanta soldati solo ottanta fanno ritorno alla base. Alla drammaticità di questo evento, si contrappone però duramente la soddisfazione dei soldati che ricevono razione doppia di viveri e sigarette, poiché lo squadrone è stato decimato: «Oggi abbiamo la pancia piena di fagioli bianchi con carne di manzo, e siamo sazi e soddisfatti [...] il furiere ricevette per il giorno del nostro ritorno il quantitativo di viveri e fece provviste per una compagnia di forza di centocinquanta uomini. Invece l'ultimo giorno si ebbe un'eccezionale sparatoria di grossi calibri e pioggia di grosse schegge [...] sicché avemmo forti perdite e ritornammo con soli ottanta uomini» (C. Abbate, N.-T. Licciardi, M. Marisaldi, A. Montebarocci, M. Romeo, C. Tiron, $\mathrm{R}$. Turco, La crudeltà della guerra rappresentata attraverso il tema del cibo in Niente di nuovo sul fronte occidentale).

Il dramma non nasce solo dal problema della quantità e qualità del cibo distribuito e della "fame " continuamente evocata nel romanzo, ma anche dai compromessi, con l'igiene e con la morale, che procurarsi da mangiare spesso comporta:

Un grosso ratto stava a cavallo sulla pagnotta. Infine decidiamo di farla finita: tagliamo via con cura i pezzi di pane che le bestiacce hanno addentato; buttar via le pagnotte non si può, si rischia di rimanere l'indomani senza mangiare [...]. Haje ha buscato anche una baguette di bianco pane francese e se l'è infilata nella cintola come una vanghetta. Veramente è un po' insanguinata a un'estremità ma non importa, si può tagliare via (ibid.).

In effetti, è stato notato, nell'opera è ricorrente il contrasto tra "cibo buono" e "cibo cattivo", laddove il valore gastronomico s'interseca con quello morale: è "buono" il cibo che non solo sostenta gli uomini, ma cementa i rapporti di solidarietà; è cibo di pura sopravvivenza quello che non ha alcun valore aggiuntivo o che è usato come merce di scambio; è infine "cattivo" quello che promuove o rappresenta simbolicamente la violenza, l'odio, l'ipocrisia (come il rancio distribuito prima della battaglia o quello provocatoriamente esibito davanti ai prigionieri affamati, o ancora la birra offerta al protagonista dai borghesi, che non hanno esitato a mandare a morire tutta un'inesperta generazione che credeva nei loro insegnamenti).

Invece, il "cibo buono" finisce con l'essere elemento d'identità sociale (tra commilitoni che lo condividono), ma anche nazionale e familiare:

"Cibo buono" è anche quello che Paolo trova nella sua casa d'origine, dove, malgrado le ristrettezze della guerra, il sabato, secondo le consuetudini, si mangiano le frittelle di patate. L'odore di frittelle che Paolo sente entrando è quindi l'odore di casa, e appena la mamma lo vede gli offre dei biscotti stantii che nelle sue intenzioni dovevano essere prelibati. Quando la licenza di Paolo è terminata e il giovane, prima di recarsi al fronte, fa tappa in un campo di istruzione, riceve dal padre e dalla sorella un pacchetto di frittelle di patate cucinategli dalla madre. Per lui, non si tratta solo di un dolce, ma quelle frittelle sono come le carezze di sua madre, 
l'espressione di una tenerezza che risulta inconcepibile in una realtà implacabile e dura come quella del fronte. Arrivato in prima linea, Paolo offre le frittelle fatte da sua madre ai suoi commilitoni e Kat riconosce subito il cibo di casa dell'amico: assaggiando le frittelle di patate cucinate dalla madre di lui, afferma che ha indovinato dal sapore la provenienza di quel dolce (G. Lazzari, C. Paolucci, L. Ricci Maccarini. Cibo e vita militare nel romanzo Niente di nuovo sul fronte occidentale di Erich Maria Remarque).

Le due opere hanno permesso di individuare sia la natura identitaria (sociale, nazionale, familiare) del cibo, sia la funzione emblematica che esso assume per gli autori. D'altra parte, dal confronto dei due libri - e anche di quelli esaminati in classe oralmente (La pelle di Malaparte e Ragazzi di vita o Una vita violenta di Pasolini) - si sono potute riconoscere con chiarezza le trasformazioni nelle abitudini alimentari, e dunque nel modo di vivere e di pensare, che la "guerra dei trent'anni" produsse rispetto ai periodi di pace che la precedettero (attestati da Sull'Oceano) e la seguirono (perlomeno nell'immagine pasoliniana delle borgate romane): neppure i più poveri degli emigranti deamicisiani paiono soffrire di quella penuria alimentare che è ben testimoniata sia da Remarque che da Malaparte, e che non pare del tutto superata neanche nell'epoca di ambientazione dei romanzi di Pasolini.

\section{I lavori individuali: gli appunti condivisi, le audio/video- interviste, i ricettari, la tesina}

Alcuni alunni hanno prodotto materiali condivisi e approfondimenti di aspetti specifici dell'argomento. La partecipazione alle due giornate di studio e la compilazione dei relativi appunti hanno costituito l'indispensabile propedeutica per l'intero lavoro. Gli studenti sono abituati a prendere appunti durante le lezioni e a servirsene per lo studio individuale, ma in questo caso l'insegnante - decidendo di farli trascrivere a dei volontari su una piattaforma online condivisa - si è posta un duplice obiettivo didattico: da un canto, gratificare le incaricate rendendole responsabili della realizzazione di un "manuale", che fosse recepito quale strumento di registrazione e trasmissione dei dati; dall'altro, monitorare le tecniche di sintesi e l'apprendimento degli studenti, rendendosi conto di quali elementi delle conferenze venivano meglio recepiti, per costruire intorno a questi il percorso didattico successivo. Va ricordata a questo proposito la grande utilità del dispositivo "Cronologia revisioni" disponibile per i documenti di Google Drive, 
che consente di seguire tutte le modifiche apportate (in questo caso dai compagni, invitati a correggere e integrare gli appunti) a un documento compilato online.

Altra idea suggerita dall'insegnante era di costituire una micro "banca della memoria", ispirata al portale bancadellamemoria.it, che «archivia esperienze di vita raccontate nel formato della videointervista da donne e uomini nati prima del 1940», quasi come risposta «a chi considera la vecchiaia un costo, un'età improduttiva» (Bajani 2008). Tale spunto, del resto, si era già dimostrato stimolante anche in una precedente esperienza didattica della docente 5 , che aveva ottenuto grande partecipazione emotiva e indotto una riflessione sul valore della memoria individuale. Sempre riconducibili all'idea della "banca della memoria" anche i due ricettari "di guerra" ricostruiti da due studentesse intervistando le nonne: documenti che, più che attestare la crisi alimentare di un determinato momento storico, dimostrano la povertà ma anche la creatività della cucina italiana, come si può dedurre dalla tipicità regionale dei piatti ricordati (panicottu, fave nette, frise, sagne ritorte, purceddhruzzi nel ricettario pugliese; tortellini ${ }^{6}$, torta di riso, torta di tagliatelline ecc. in quello bolognese, dove spiccano le ricette dei "surrogati" pappa matta e caffè fatto con foglie di cicoria).

Va segnalato l'interesse del "menù settimanale" rievocato dalla signora Ida Busi Bartolini e schematizzato dalla nipote, Letizia Ricci Maccarini, da cui emerge non solo una dieta costituita da un piatto "unico" a pasto, ma la netta predominanza di carboidrati e legumi (proteine vegetali), a fronte dell'assenza di proteine animali (come si ricava da entrambi i ricettari):

\begin{tabular}{|l|l|l|l|}
\hline $\begin{array}{l}\text { Giorni della } \\
\text { Settimana }\end{array}$ & Colazione & Pranzo & Cena \\
\hline Lunedì & $\begin{array}{l}\text { Caffellatte con pane } \\
\text { raffermo }\end{array}$ & Pasta in brodo + un frutto & Fagioli in "umido" \\
\hline Martedì & $\begin{array}{l}\text { Caffellatte con pane } \\
\text { raffermo }\end{array}$ & $\begin{array}{l}\text { Pasta asciutta condita con } \\
\text { burro, pomodoro o ragù + } \\
\text { un frutto }\end{array}$ & $\begin{array}{l}\text { Riso con le patate o riso } \\
\text { cotto nel latte }\end{array}$ \\
\hline Mercoledì & $\begin{array}{l}\text { Caffellatte con pane } \\
\text { raffermo }\end{array}$ & $\begin{array}{l}\text { Zuppa o pasta con i fagioli + } \\
\text { un frutto }\end{array}$ & $\begin{array}{l}\text { Caffellatte e pane } \\
\text { raffermo }\end{array}$ \\
\hline
\end{tabular}

Una video-intervista a un nonno partigiano, vincitrice nel 2011 di un premio ANPI: cf. http://cineletteratura4d.blogspot. it/2011/05/ecco-il-video-vincitore.html.

Peraltro, la presenza dei tortellini in un "menù di guerra" potrebbe spiegarsi non solo come fatto eccezionale, legato a festività o particolare benessere dell'intervistata, se si considera quest'altra testimonianza: «[...] i tortellini che ti aspettavano a casa. Vuoti. Li chiamavano matti, ma era buoni; qualcuno sosteneva anche meglio dei veri perché quelli, almeno, c'erano, mentre degli altri si era persa financo la memoria» (Ferrari 1980, p. 30). 


\begin{tabular}{|l|l|l|l|}
\hline $\begin{array}{l}\text { Giorni della } \\
\text { Settimana }\end{array}$ & Colazione & Pranzo & Cena \\
\hline Giovedi & $\begin{array}{l}\text { Caffellatte con pane } \\
\text { raffermo }\end{array}$ & $\begin{array}{l}\text { Pasta asciutta condita con } \\
\text { burro, pomodoro o ragù + } \\
\text { un frutto }\end{array}$ & Fagioli in "umido" \\
\hline Venerdi & $\begin{array}{l}\text { Caffellatte con pane } \\
\text { raffermo }\end{array}$ & $\begin{array}{l}\text { Zuppa o pasta con i fagioli + } \\
\text { un frutto }\end{array}$ & $\begin{array}{l}\text { Riso con le patate o riso } \\
\text { cotto nel latte }\end{array}$ \\
\hline Sabato & $\begin{array}{l}\text { Caffellatte con pane } \\
\text { raffermo }\end{array}$ & $\begin{array}{l}\text { Pasta asciutta condita con } \\
\text { burro, pomodoro o ragù + } \\
\text { un frutto }\end{array}$ & $\begin{array}{l}\text { Caffellatte e pane } \\
\text { raffermo }\end{array}$ \\
\hline Domenica & $\begin{array}{l}\text { Caffellatte con pane } \\
\text { raffermo }\end{array}$ & Pasta in brodo + un frutto & $\begin{array}{l}\text { Riso con le patate o riso } \\
\text { cotto nel latte }\end{array}$ \\
\hline
\end{tabular}

La tesina, infine, individua uno dei nodi cruciali, nell'interesse dei giovani, del problema dell'alimentazione dal passato ad oggi:

si è passati in meno di un secolo dal non possedere quasi nulla da mangiare, al possederne troppo. Il fatto è che nessuno dei due elementi è positivo. Tutti e due causano malnutrizione, che a sua volta è portatrice di gravi malattie o persino della morte di un individuo (G. Bernardi, cit).

Nell'osservazione di elementi che rivelino questa tensione dialettica, che suggella la complessità e la pluralità della cultura odierna, si può, forse, individuare la meta del nostro percorso.

\section{Bibliografia}

Anselmi G. M. e Ruozzi G. (eds.) 2011, Banchetti letterari, Roma: Carocci.

Bajani A. 2008, Youtube della terza età, "Il Sole 24 ore", 17 luglio.

Baroncini D. 2011, Cucina futurista, in Anselmi e Ruozzi (eds.) 2011, Banchetti letterari, Roma: Carocci, 166-171.

Barthes R. 1974, Miti d'oggi, Torino: Einaudi (ed. or. 1957).

Bonazzi N. 2009, La letteratura carnevalesca, in Raimondi, Anselmi, Chines e Fenocchio (eds.) 2009, Leggere, come io l'intendo. Umanesimo e Rinascimento, vol. 2, Milano: Bruno Mondadori, 514-541.

Capaci B. 2011, Pasta e maccheroni, in Anselmi e Ruozzi (eds.) 2011, Banchetti letterari, Roma: Carocci, 254-261.

De Amicis E. 1996, Sull'oceano, Milano: Garzanti (ed. or. 1889).

Ferrari A. 1980, Quando la dieta era più ferrea della volontà, in Goldoni, Ferrari e Leoni (eds.) 1980, I giorni di Bologna Kaputt, Bologna: Edizioni Giornalisti associati, 27-39.

Fischler C. 1997, La «macdonaldizzazione» dei costumi, in Flandrin e Montanari (eds.) 1997, Storia dell'alimentazione, Roma-Bari: Laterza, 679-695. 
Flandrin J.-L. e Montanari M. (eds.) 1997, Storia dell'alimentazione, Roma-Bari: Laterza.

Gilli L. 2016, Riflessioni sull'identità nella narrativa gastronomica e nell'arte del cibo, "Centro Studi Camporesi", http://www.griseldaonline.it/camporesi/cibo/riflessioninarrativa-gastronomica-laura-gilli.html

Goldoni L., Ferrari A. e Leoni G. (eds.) 1980, I giorni di Bologna Kaputt, Bologna: Edizioni Giornalisti associati.

Malaparte C. 1959, La pelle, Firenze: Vallecchi (ed. or. 1949).

Marinetti F. T. 1930, Manifesto della cucina futurista, "Gazzetta del Popolo di Torino", 28 agosto.

Meldini P. 1997, L'emergere delle cucine regionali: l'Italia, in Flandrin e Montanari 1997, Storia dell'alimentazione, Roma-Bari: Laterza, 658-664.

Montanari M. 1991, Nuovo convivio. Storia e cultura dei piaceri della tavola nell'età moderna, Roma-Bari: Laterza.

Montanari M. 1993, La fame e l'abbondanza. Storia dell'alimentazione in Europa, Roma-Bari: Laterza.

Montanari M. 2002, Il mondo in cucina. Storia, identità,scambi, Roma-Bari: Laterza.

Montanari M. 2004, Il cibo come cultura, Roma-Bari: Laterza.

Montanari M. 2009, Il riposo della polpetta, Roma-Bari: Laterza.

Montanari M. 2010, L'identità italiana in cucina, Roma-Bari: Laterza.

Pasolini P.P. 1955, Ragazzi di vita, Milano: Garzanti.

Pasolini P.P. 1959, Una vita violenta, Milano: Garzanti.

Portincasa A. 2016, Scrivere di gusto. Una storia della cucina italiana attraverso i ricettari, Bologna: Pendragon.

Raimondi E., Anselmi G. M., Chines L. e Fenocchio G. (eds.) 2009, Leggere, come io l'intendo. Umanesimo e Rinascimento, vol. 2, Milano: Bruno Mondadori.

Remarque E. M. 1989, Niente di nuovo sul fronte occidentale, Milano: Mondadori (ed. or. 1929).

Ruozzi C. 2011, Cibi di guerra, in Anselmi e Ruozzi (eds.) 2011, Banchetti letterari, Roma: Carocci, 108-117.

Soglia S. 1980, E fu subito pane nero, in Goldoni, Ferrari e Leoni (eds.) 1980, I giorni di Bologna Kaputt, Bologna: Edizioni Giornalisti associati, 40-42.

Spurlock M. 2005, Non mangiate questo libro: il fast food e l'America super size, Roma: Fandango.

Varotti C. 2011, Abbuffata, in Anselmi e Ruozzi (eds.) 2011, Banchetti letterari, Roma: Carocci, 13-22. 


\section{Risorse on line}

Guerrainfame. La guerra non è mai servita. Cibi di guerra, strategie e politiche alimentari nella prima metà del Novecento in Italia

http://guerrainfame.it/

Lavori realizzati dalla classe V H ESABAC 2015-16 del Liceo "Laura Bassi” di Bologna

http://guerrainfame.it/classe_5_h_esabac_del_liceo_laura_bassi_di_bologna_2015-2016. 